\title{
Instabilities leading to vortex lattice formation in rotating Bose-Einstein condensates
}

\author{
N. G. Parker, ${ }^{1}$ R. M. W. van Bijnen, ${ }^{1,2}$ and A. M. Martin ${ }^{1}$ \\ ${ }^{1}$ School of Physics, University of Melbourne, Parkville, Victoria 3010, Australia \\ ${ }^{2}$ Eindhoven University of Technology, P.O. Box 513, 5600 MB Eindhoven, The Netherlands
}

(Received 6 April 2006; published 20 June 2006)

\begin{abstract}
We present a comprehensive theoretical study of vortex lattice formation in atomic Bose-Einstein condensates confined by a rotating elliptical trap. We consider rotating solutions of the classical hydrodynamic equations and their response to perturbations, as well as time-dependent simulations. We discriminate three distinct, experimentally testable, regimes of instability: ripple, interbranch, and catastrophic. Under symmetrybreaking perturbations these instabilities lead to lattice formation even at zero temperature. While our results are consistent with previous theoretical and experimental results, they shed further light on lattice formation.
\end{abstract}

DOI: 10.1103/PhysRevA.73.061603

PACS number(s): 03.75.Lm, 03.75.Kk

Vortex lattices are a striking manifestation of superfluidity in rotating systems, which have been recently generated in atomic Bose-Einstein condensates (BECs) via rotation in an elliptical harmonic trap [1-3]. Vortex lattices form for trap rotation frequencies $\Omega \sim 0.7 \omega_{\perp}$, where $\omega_{\perp}$ is the mean harmonic trap frequency in the rotating plane. Although a vortex lattice is thermodynamically favorable for much lower rotation frequencies, the instabilities necessary to seed lattice formation are only present in this region. Such instabilities have been predicted by hydrodynamic studies of condensate solutions in the rotating frame [4] and the dynamical perturbations of these states [5], and have also been analyzed in dipolar BECs [6]. Although numerical simulations of the Gross-Pitaevskii equation have also observed vortex lattice formation in this region [7-11], the results are mixed: for $\Omega \lesssim 0.7 \omega_{\perp}$, Refs. [7-9] require thermal effects to enable vortex nucleation, while Ref. [10] does not; for $\Omega \geqslant 0.7 \omega_{\perp}$, Refs. [7-11] observe a shape instability before nucleating vortices even in the absence of thermal effects. This is consistent with experiments $[3,12]$ that indicate that lattice formation is temperature independent. Breaking the rotational symmetry of such simulations has been shown to be crucial to enable realistic nucleation ofvortices [11].

In this Rapid Communication we present a thorough investigation of the instabilities leading to vortex lattice formation in elliptical traps in the regime $\Omega \leq \omega_{\perp}$. We consider the adiabatic introduction of trap ellipticity or rotation frequency. In order to probe the condensate instabilities we consider the classical hydrodynamic solutions in the rotating frame and their response to dynamical perturbations. Furthermore, we perform time-dependent (TD) simulations to test these results and probe the fate of the unstable condensate.

In the experiments $[1,3]$ the harmonic confining trap is transformed radially into an ellipse and rotated about the $z$ axis. In the limit of zero temperature, the condensate can be approximated by a mean-field "wave function" $\psi$, which can be expressed as $\psi=\sqrt{\rho} e^{i \phi}$, where $\rho$ and $\phi$ are the condensate density and phase. In the frame rotating at $\Omega$, the density $\rho$ and fluid velocity $\mathbf{v}=(\hbar / m) \boldsymbol{\nabla} \phi[13]$ satisfy the hydrodynamic equations

$$
\frac{\partial \rho}{\partial t}+\nabla \cdot[\rho(\mathbf{v}-\mathbf{\Omega} \times \mathbf{r})]=0,
$$

$$
\begin{gathered}
m \frac{\partial \mathbf{v}}{\partial t}+\nabla\left(\frac{1}{2} m \mathbf{v} \cdot \mathbf{v}+\frac{1}{2} m\left(\omega_{x}^{2} x^{2}+\omega_{y}^{2} y^{2}+\omega_{z}^{2} z^{2}\right)\right. \\
\left.+\rho g-\frac{\hbar^{2}}{2 m} \frac{\nabla^{2} \sqrt{\rho}}{\sqrt{\rho}}-m \mathbf{v} \cdot[\mathbf{\Omega} \times \mathbf{r}]\right)=0 .
\end{gathered}
$$

Here $m$ is the atomic mass, $g=4 \pi \hbar^{2} a / m$ is the interaction coefficient where $a$ is the $s$-wave scattering length (in this work $g>0$ ), and $\omega_{x}, \omega_{y}$, and $\omega_{z}$ are the harmonic trap frequencies. When the trap is elliptical we can write $\omega_{x}$ $=\sqrt{1-\epsilon} \omega_{\perp}$ and $\omega_{y}=\sqrt{1+\epsilon \omega_{\perp}}$, where $\epsilon$ characterizes the trap ellipticity, and $\omega_{\perp}^{2}=\left(\omega_{x}^{2}+\omega_{y}^{2}\right) / 2$.

Following Recati et al. [4], we employ the Thomas-Fermi (TF) approximation by neglecting the $\left(\nabla^{2} \sqrt{\rho} / \sqrt{\rho}\right)$ term in Eq. (2), and assume an irrotational quadrupolar velocity field [14],

$$
\mathbf{v}=\alpha \boldsymbol{\nabla}(x y),
$$

where $\alpha$ is the velocity field amplitude. Substituting this into Eq. (2) and setting $\partial \rho / \partial t=\partial \mathbf{v} / \partial t=0$, we obtain stationary density solutions of the form

$$
\rho_{0}=\frac{1}{g}\left(\mu-\frac{1}{2} m\left(\widetilde{\omega}_{x}^{2} x^{2}+\widetilde{\omega}_{y}^{2} y^{2}+\omega_{z}^{2} z^{2}\right)\right),
$$

in the region where the chemical potential $\mu \geq m\left(\widetilde{\omega}_{x}^{2} x^{2}\right.$ $\left.+\widetilde{\omega}_{y}^{2} y^{2}+\omega_{z}^{2} z^{2}\right) / 2$, and $\rho_{0}=0$ elsewhere. The rotation induces effective trap frequencies $\widetilde{\omega}_{x}^{2}=\left[(1-\epsilon)+\alpha^{2}-2 \alpha \Omega\right] \omega_{\perp}^{2}$ and $\widetilde{\omega}_{y}^{2}$ $=\left[(1+\epsilon)+\alpha^{2}+2 \alpha \Omega\right] \omega_{\perp}^{2}$, where $\alpha$ determines the ellipticity of the BEC density [4]. Plugging Eq. (4) into Eq. (1) we obtain [4]

$$
\widetilde{\omega}_{x}^{2}(\alpha+\Omega)+\widetilde{\omega}_{y}^{2}(\alpha-\Omega)=0
$$

for the rotating TF condensate solutions.

Figure 1(a) shows the stationary solutions in $\Omega-\alpha$ space for various values of trap ellipticity $\epsilon$. In the limit of $\epsilon=0$ (solid line) a nonrotating $(\alpha=0)$ solution occurs for all $\Omega$, with two additional solutions bifurcating from the $\alpha=0$ axis at $\Omega_{\text {bif }}^{0}=\omega_{\perp} / \sqrt{2}$. For finite $\epsilon$ (dotted and dashed lines), the $\alpha=0$ solution disappears and the plot consists of two distinct branches. The upper branch (positive $\alpha$ ) is single valued and exists for all $\Omega$, while the lower branch (negative $\alpha$ ) is double valued and exists only when $\Omega$ is greater than the 


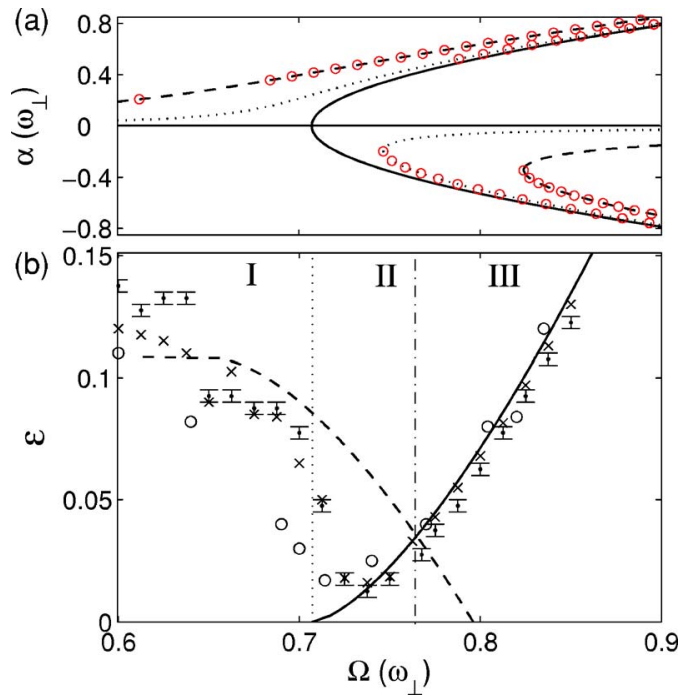

FIG. 1. (Color online) (a) Velocity field amplitude $\alpha$ of the stationary solutions of Eq. (5) versus rotation frequency $\Omega$ for $\epsilon$ $=0$ (solid line), 0.02 (dotted line), and 0.1 (dashed line). Regions of dynamical instability for $\epsilon=0.02$ and 0.1 are shown (circles). (b) Phase diagram of $\epsilon$ versus $\Omega$. Plotted are the bifurcation point $\Omega_{\text {bif }}(\epsilon)$ (solid line) from Eq. (5), the onset of dynamical instability $\Omega_{\text {ins }}(\epsilon)$ from Eq. (6) (dashed line), and experimental data of Hodby et al. [3] (circles). Simulations of Eqs. (1) and (2) (with $\mu$ $\left.=10 \hbar \omega_{\perp}\right)$ show the critical ellipticities beyond which the condensate deviates from an elliptical shape $\epsilon_{\mathrm{cr}}^{\mathrm{dev}}$ (crosses) and beyond which lattice formation ultimately occurs $\epsilon_{\mathrm{cr}}^{\text {inst }}$ (points with error bars). The bifurcation point for $\epsilon=0$ (dotted line) and the crossing frequency $\Omega_{\mathrm{X}}$ (dot-dashed line) at which $\Omega_{\mathrm{bif}}(\epsilon)=\Omega_{\mathrm{ins}}(\epsilon)$ are indicated.

bifurcation frequency $\Omega_{\text {bif }}(\epsilon)$. As $\epsilon$ is increased from zero, the branches move away from the $\alpha=0$ axis [Fig. 1(a)]. Furthermore the bifurcation point of the lower branch $\Omega_{\text {bif }}(\epsilon)$ shifts to higher $\Omega$, as shown in Fig. 1(b) (solid line). The branch diagrams are key to understanding the response of the system to the adiabatic introduction of trap ellipticity $\epsilon$ or rotation frequency $\Omega[2]$. Before any rotation and ellipticity is applied the BEC has $\alpha=0$. When $\Omega$ is increased adiabatically (for fixed $\epsilon$ ), the BEC follows the upper branch, with increasing $\alpha$. When $\epsilon$ is introduced adiabatically (for fixed $\Omega)$, the BEC can follow two routes, depending on the value of $\Omega$ relative to the bifurcation point $\Omega_{\text {bif }}^{0}$ [dotted line in Fig. 1(b)]. For $\Omega<\Omega_{\text {bif }}^{0}$, the lower branch is nonexistent and the BEC follows the upper branch to increasing values of $\alpha$. For $\Omega>\Omega_{\text {bif }}^{0}$, the BEC follows the lower branch from $\alpha=0$ to negative $\alpha$. However, as $\epsilon$ is increased, the edge of the lower branch $\Omega_{\text {bif }}(\epsilon)$ shifts to higher frequencies, and when $\Omega_{\text {bif }}(\epsilon)>\Omega$ the lower branch no longer exists. In this manner, the evolution of the branches can induce instability, and has been linked to lattice formation [2].

The stationary solutions of Eq. (5) are not necessarily stable solutions. To investigate their stability we follow Sinha and Castin [5] by considering small perturbations $\delta \rho$ and $\delta \phi$ of stationary solutions of density $\rho_{0}$ and phase $\phi_{0}$. Taking variational derivatives of Eqs. (1) and (2) we obtain the time evolution equations

$$
\frac{\partial}{\partial t}\left[\begin{array}{c}
\delta \phi \\
\delta \rho
\end{array}\right]=-\left[\begin{array}{cc}
\mathbf{v}_{c} \cdot \boldsymbol{\nabla} & g / m \\
\boldsymbol{\nabla} \cdot\left(\rho_{0} \boldsymbol{\nabla}\right) & \mathbf{v}_{c} \cdot \boldsymbol{\nabla}
\end{array}\right]\left[\begin{array}{c}
\delta \phi \\
\delta \rho
\end{array}\right]
$$

where $\mathbf{v}_{c}=\mathbf{v}-\boldsymbol{\Omega} \times \mathbf{r}$ is the velocity field in the rotating frame. The eigenfunctions of Eq. (6) grow in time as $e^{\lambda t}$, where $\lambda$ is the eigenvalue. Solutions are unstable when one or more eigenvalues contain a positive real part, and are thought to seed vortex lattice formation [5].

Using a polynomial ansatz for the perturbations, this predicts dynamical instability when $\Omega$ exceeds a critical value $\Omega_{\text {ins }}(\epsilon)$ [5] [circles in Fig. 1(a)]. The unstable modes have length scales of the order of the BEC size, much greater than the healing length $\xi=\hbar / \sqrt{m n_{0} g}$ that characterizes the vortex size $\left(n_{0}\right.$ is the peak density). In the limit of $\epsilon=0, \Omega_{\text {ins }}$ $\approx 0.78 \omega_{\perp}$. As $\epsilon$ is increased, $\Omega_{\text {ins }}(\epsilon)$ is reduced [dashed line in Fig. 1(b)]. The outlying point in Fig. 1(a) for $\epsilon=0.1$ (dashed line) at $\Omega \approx 0.61 \omega_{\perp}$ is not considered to be in the unstable region due to its narrow width and small eigenvalues [16]. Note that the lower-branch solution closest to the $\alpha=0$ axis is not dynamically unstable. A key rotation frequency in our work is $\Omega_{\mathrm{X}}$, which is the crossing point of $\Omega_{\text {bif }}(\epsilon)$ and $\Omega_{\text {ins }}(\epsilon)$, and has the value $\Omega_{\mathrm{X}} \approx 0.765 \omega_{\perp}$ [dotdashed line in Fig. 1(b)].

From the solutions of Eq. (5) and the dynamical instability of Eq. (6) we can predict the BEC stability following the adiabatic introduction of $\Omega$ or $\epsilon$. However, to determine how the instability manifests itself and if it leads to lattice formation we perform TD simulations of Eqs. (1) and (2) in the laboratory frame. This is equivalent to solving the GrossPitaevskii equation [11]. Following previous approaches [8-11], and noting that the solutions of Eqs. (5) and (6) are independent of $z$ [17], we consider a two-dimensional system. The initial state is found by imaginary-time propagation. Then, in real time, either $\epsilon$ is ramped up linearly at a rate $d \epsilon / d t=10^{-4} \omega_{\perp}$ (for fixed $\Omega$ ), or $\Omega$ is ramped up linearly at a rate $d \Omega / d t=10^{-2} \omega_{\perp}^{2}$ (for fixed $\epsilon$ ). We derive $\alpha$ by fitting the velocity field in a central region $3 \times 3 \mu \mathrm{m}$ to the form of Eq. (3).

In such "idealized" simulations the trap and BEC can maintain a twofold rotational symmetry to unrealistic levels. This strongly inhibits vortex nucleation since the vortices must enter in pairs. Symmetry breaking, rather than thermal effects, has been shown to be crucial in simulating lattice formation [11]. Indeed, Eq. (6) predicts that only odd modes are dynamically unstable. While previous studies [7,9] have broken this symmetry through the introduction of thermal "noise," we shift the trap by $0.5 \xi$ before running in real time in order to excite odd modes [15].

We first consider the adiabatic increase of trap ellipticity $\epsilon$ for fixed $\Omega$. We discriminate three cases of instability as indicated in Fig. 1(b): (I) ripple, (II) interbranch, and (III) catastrophic instabilities.

(I) Ripple instability $\Omega<\omega_{\perp} / \sqrt{2}$. The case for $\Omega$ $=0.7 \omega_{\perp}$ is shown in Fig. 2(a)I. The velocity field amplitude $\alpha$ (dots) follows the upper branch of Eq. (5) (solid line). Here the BEC and trap axes rotate in phase, as noted experimentally $[2,3]$. As $\epsilon$ is increased, the BEC moves along the branch to higher $\alpha$, and when $\epsilon$ exceeds a critical value (corresponding to when $\Omega>\Omega_{\text {inst }}$ ) the solution becomes dynami- 

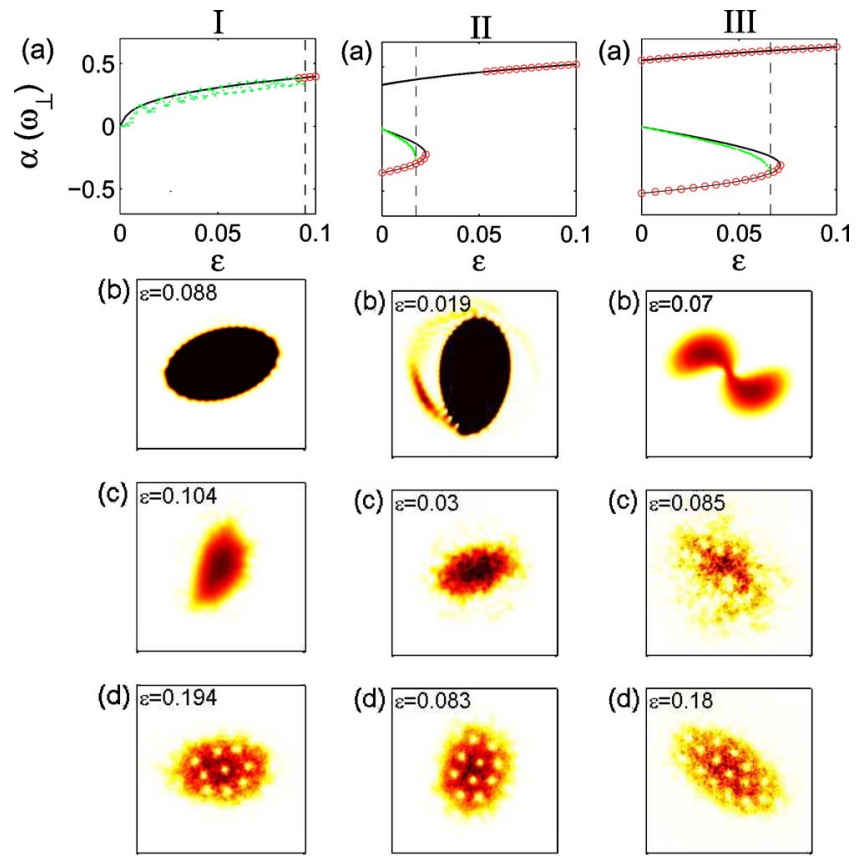

FIG. 2. (Color online) Dynamics under a continuous increase of $\epsilon$ (at a rate of $\left.d \epsilon / d t=10^{-4} \omega_{\perp}\right)$ for (I) ripple instability $\left(\Omega / \omega_{\perp}\right.$ $=0.7)$, (II) interbranch instability $\left(\Omega / \omega_{\perp}=0.75\right)$, and (III) catastrophic instability $\left(\Omega / \omega_{\perp}=0.8\right)$. (a) Velocity field amplitude $\alpha$ versus $\epsilon$ according to Eq. (5) [(black) solid lines] and TD simulations of Eqs. (1) and (2) [(green) dots]. To the right of the dashed line, Eq. (3) is no longer a valid fit to the simulated velocity field (the standard deviation of the fit becomes of the order of $\alpha$ ). The regions of dynamical instability are indicated [(red) circles]. Density snapshots showing the (b) onset of instability, (c) disrupted state seeded by instability, and (d) vortex lattice. Dark (light) regions represent high (low) density. Each box represents a region $12 \times 12 \mu \mathrm{m}=(20$ $\times 20) a_{\perp}$, where $a_{\perp}=\left(\hbar / m \omega_{\perp}\right)^{1 / 2}$ is the mean harmonic oscillator length (the simulation box is much greater than this). In (Ib) and (IIb) the density scale is limited to $0.1 \% n_{0}$ to highlight low-density features.

cally unstable, according to Eq. (6). This occurs for $\epsilon$ $\approx 0.09$ in Fig. 2(a)I (dashed line). Subsequently $\alpha$ (dots) deviates from the upper branch (solid lines), consistent with the onset of dynamical instability. For low $\epsilon, \alpha$ (dots) oscillates slightly due to the center-of-mass motion caused by the initial offset of the BEC.

At a critical ellipticity $\epsilon_{\mathrm{cr}}^{\mathrm{dev}}$ low-density ripples form on the BEC edge [Fig. 2(b)I], each on the scale of the healing length and with a phase singularity ("ghost" vortices [7-9]), and grow in amplitude as $\epsilon$ is increased. If $\epsilon$ becomes fixed when the ripples have very low amplitude, they do not grow over the time scales considered. However, once $\epsilon$ exceeds a second critical value $\epsilon_{\mathrm{cr}}^{\text {ins }}$ (corresponding to when the ripple amplitude is of order of $10 \% n_{0}$ ), the dynamical instability of Eq. (6) is triggered by the ripples. This instability generates large-scale shape oscillations [Fig. 2(c)I], disrupting the $\mathrm{BEC}$, and enabling ghost vortices to nucleate, which slowly crystallize into a lattice [Fig. 2(d)I] [11]. Once $\epsilon_{\mathrm{cr}}^{\text {ins }}$ is reached, lattice formation occurs independently of whether $\epsilon$ is fixed or continuously increased. Since the ripples originate in the non-TF tails of the BEC, they cannot be explained by the TF analytics of Eqs. (3)-(6), and a non-TF approach would be required to explain their origin.

Surface ripples have been observed experimentally to precede vortex nucleation at this $\Omega$ [3]. The gradual growth of the ripples leads to a slow injection of energy or angular momentum into the system, as observed elsewhere at similar $\Omega[7,10]$. Note that according to Eq. (6) the dynamical instability on the upper branch couples to odd modes only. If the symmetry is preserved we do not expect the instability to develop [7-9].

(II) Interbranch instability $\omega_{\perp} / \sqrt{2}<\Omega<\Omega_{\mathrm{X}}$. The case for $\Omega=0.75 \omega_{\perp}$ is shown in Fig. 2(a)II. Since $\Omega>\omega_{\perp} / \sqrt{2}, \alpha$ (dots) initially follows the lower-branch solutions, where we observe that the BEC and trap axes are $\pi / 2$ out of phase. As $\epsilon$ is increased, a point is reached when $\Omega<\Omega_{\text {bif }}(\epsilon)$. Here the lower branch is no longer a solution of Eq. (5). In Fig. 2(a)II, this occurs for $\epsilon \approx 0.02$ (dashed line). Due to the non-TF nature of the numerical solutions of Eqs. (1) and (2), $\alpha$ does not perfectly fit the branch solutions of Eq. (5).

When $\alpha$ (dots) reaches the cusp of the lower branch it deviates nonadiabatically, as observed experimentally [2]. Since $\Omega<\Omega_{X}$, the upper branch is dynamically stable at this $\epsilon$. The BEC tries to transform to the upper branch, but without dissipation $\alpha$ (dots) is forced to oscillate between positive and negative values, with the density oscillating between a circular and highly elliptical shape. If $\epsilon$ is fixed close to this critical ellipticity, the shape oscillations are stable. However, if $\epsilon$ is increased further, the shape oscillations destabilize, with the BEC shedding low-density material at its extrema in a spiral pattern [Fig. 2(b)II]. This situation is closely analogous to when $\Omega$ is suddenly turned on, with the fate of the BEC being qualitatively similar [11]. The growth of the ejected material gradually destabilizes the BEC [Fig. 2(c)II], leading to vortex nucleation and ultimately a lattice [Fig. 2(c)II]. This is consistent with the observations in [2].

(III) Catastrophic instability $\Omega>\Omega_{\mathrm{X}}$. The case for $\Omega$ $=0.8 \omega_{\perp}$ is shown in Fig. 2(a)III. Again $\alpha$ (dotted line) follows the lower branch, which ceases to be a solution at some critical $\epsilon$ (dashed line). However, since $\Omega>\Omega_{\mathrm{X}}$, the upper branch is dynamically unstable and no stable solutions exist. The BEC undergoes a quick and catastrophic instability, with $\alpha$ (dots) deviating rapidly from the rotating solutions of Eq. (5). The BEC density becomes strongly contorted into a spiral shape [Fig. 2(b)III]. The arms of the spiral collapse inward and trap phase singularities to form vortices. Energy and angular momentum are very rapidly injected into the BEC (in contrast to the ripple and interbranch instabilities), as observed in $[7,10]$ in this frequency regime. The BEC becomes highly excited and turbulent [Fig. 2(c)III], with structure on length scales less than the healing length. Although we observe this state to ultimately settle into a lattice [Fig. 2(d)III], one may question the validity of our zerotemperature simulations for such a "heated" state. Indeed, for $\Omega \gtrsim 0.78 \omega_{\perp}$, turbulent states, rather than vortex lattices, were observed experimentally in [1].

In the TD simulations we have measured two distinct critical ellipticites: $\epsilon_{\mathrm{cr}}^{\mathrm{dev}}$ [crosses in Fig. 1(b)] is when, for a continuously increasing $\epsilon$, we observe the density to deviate from a smooth ellipse (on the level of $0.1 \% n_{0}$ ); $\epsilon_{\mathrm{cr}}^{\text {inst }}$ [points with error bars in Fig. 1(b)] is when, for $\epsilon$ ramped up to some 
nal value, instability and lattice formation ultimately occur. In regime I, typically $\epsilon_{\mathrm{cr}}^{\mathrm{dev}} \leq \epsilon_{\mathrm{cr}}^{\text {inst }}$ since surface ripples are generated that are stable for a narrow range of $\epsilon$, above which they induce instability and lattice formation. In regimes II and III, $\epsilon_{\mathrm{cr}}^{\mathrm{dev}} \approx \epsilon_{\mathrm{cr}}^{\mathrm{ins}}$, indicating the relatively sudden onset of this instability once the density deviates from a smooth ellipse.

According to the TD simulations, the region above the points in Fig. 1(b) is unstable, leading to lattice formation. The prediction of Eq. (6) (dashed line) gives reasonable agreement with the TD results in region I, while Eq. (5) gives excellent agreement in region III. Also plotted in Fig. 1(b) are the experimental results of Hodby et al. [3] (circles). The TD results give good agreement with the experimental data throughout, with the agreement being particularly good in region III.

So far we have considered the adiabatic introduction of $\epsilon$ for fixed $\Omega$. However, Fig. 1(b) also applies to the case when $\Omega$ is introduced adiabatically for fixed $\epsilon$. Here the BEC follows the upper branch until it becomes dynamically unstable to the ripple instability. According to Eq. (6) dynamical instability occurs when $\Omega>\Omega_{\text {inst }}(\epsilon)$ [dashed line in Fig. 1(b)]. In [2], for $\epsilon=0.025$, vortex lattice formation was observed to occur when $\Omega \gtrsim 0.75 \omega_{\perp}$, which agrees well with our TD simulations and Fig. 1(b).

Equations (5) and (6) are valid in the TF limit of $\mu / \hbar \omega_{\perp} \gg 1$. For the non-TF simulations of Eqs. (1) and (2) we have employed an intermediate value of $\mu / \hbar \omega_{\perp}=10$. We find that for less (more) Thomas-Fermi-like condensates, the TD results in Fig. 1(b) shift downward (upward), deviating away from (toward) the TF predictions of Eqs. (5) and (6). In addition, as $\mu / \hbar \omega_{\perp}$ is increased, the TD values of $\alpha$ in Fig. 2(a)II and 2(a)III (dots) become closer to the branch solutions (solid lines).

We have shown that vortex lattice formation is inherently a two-dimensional and zero-temperature effect (although finite-temperature effects may speed up the crystallization process). We have theoretically mapped out the condensate stability as a function of rotation frequency $\Omega$ and trap ellipticity $\epsilon$, with our interpretation being consistent with previous experimental and theoretical results. Specifically, for fixed $\Omega$ and adiabatic introduction of $\epsilon$ we find three distinct regimes of instability-ripple $\left(\Omega<\omega_{\perp} / \sqrt{2}\right)$, interbranch $\left(\omega_{\perp} / \sqrt{2}<\Omega<\Omega_{\mathrm{X}}\right)$, and catastrophic $\left(\Omega>\Omega_{\mathrm{X}}\right)$. Each instability manifests itself in a characteristic manner (which could be observed experimentally) and ultimately seeds vortex lattice formation. This formation process not only relies on the presence of an instability but is crucially dependent on the breaking of the twofold rotational symmetry of the system, as inevitably occurs experimentally.

We acknowledge support from the ARC and the University of Melbourne. We thank Y. Castin, C. S. Adams, and C. J. Foot for helpful comments, and C. J. Foot for the use of experimental data.
[1] K. W. Madison, F. Chevy, W. Wohlleben, and J. Dalibard, Phys. Rev. Lett. 84, 806 (2000).

[2] K. W. Madison, F. Chevy, V. Bretin, and J. Dalibard, Phys. Rev. Lett. 86, 4443 (2001).

[3] E. Hodby et al., Phys. Rev. Lett. 88, 010405 (2001).

[4] A. Recati, F. Zambelli, and S. Stringari, Phys. Rev. Lett. 86, 377 (2001).

[5] S. Sinha and Y. Castin, Phys. Rev. Lett. 87, 190402 (2001).

[6] R. M. W. van Bijnen, D. H. J. O’Dell, N. G. Parker, and A. M. Martin, e-print cond-mat/0602572.

[7] C. Lobo, A. Sinatra, and Y. Castin, Phys. Rev. Lett. 92, 020403 (2004).

[8] M. Tsubota, K. Kasamatsu, and M. Ueda, Phys. Rev. A 65 , 023603 (2002); K. Kasamatsu, M. Tsubota, and M. Ueda, ibid. 67, 033610 (2003).

[9] A. A. Penckwitt, R. J. Ballagh, and C. W. Gardiner, Phys. Rev.
Lett. 89, 260402 (2002).

[10] E. Lundh, J.-P. Martikainen, and K.-A. Suominen, Phys. Rev. A 67, 063604 (2003).

[11] N. G. Parker and C. S. Adams, Phys. Rev. Lett. 95, 145301 (2005); J. Phys. B 39, 43 (2006).

[12] J. R. Abo-Shaeer, C. Raman, and W. Ketterle, Phys. Rev. Lett. 88, 070409 (2002).

[13] This is the fluid velocity $\mathbf{v}$ in the laboratory frame, expressed in rotating frame coordinates.

[14] Y. Castin and R. Dum, Eur. Phys. J. D 7, 399 (1999).

[15] Over long times, symmetry can break intrinsically through the stimulated growth of numerical noise [11].

[16] Numerical simulations do not indicate any dynamical instability within these narrow, low-eigenvalue regions.

[17] As in [5] we find that the regions of instability according to Eq. (6) are independent of $\omega_{z}$. 